\title{
Clinical and epidemiologic profile of the initial COVID-19 patients at a tertiary care centre in India
}

\author{
Nitesh Gupta1, Sumita Agrawal ${ }^{2}$, Pranav Ish ${ }^{1}$, Suruchi Mishra ${ }^{3}$, Rajni Gaind ${ }^{4}$, Ganapathy Usha ${ }^{5}$, \\ Balvinder Singh ${ }^{6}$, Manas Kamal Sen ${ }^{1}$ for the Safdarjung Hospital COVID 2019 working group* \\ ${ }^{1}$ Department of Pulmonary, Critical Care and Sleep Medicine, Vardhman Mahavir Medical College and Safdarjung Hospital, \\ New Delhi; ${ }^{2}$ Department of Pulmonary Medicine, Medipulse Hospital, Jodhpur; ${ }^{3}$ Department of Community Medicine, \\ Vardhman Mahavir Medical College and Safdarjung Hospital, New Delhi; ${ }^{4}$ Department of Microbiology, Vardhman Mahavir \\ Medical College and Safdarjung Hospital, New Delhi; ${ }^{5}$ Department of Anaesthesiology, Vardhman Mahavir Medical College \\ and Safdarjung Hospital, New Delhi; ${ }^{6}$ Medical Superintendent, Safdarjung Hospital, New Delhi, India
}

\begin{abstract}
COVID-19 has now become a pandemic. It has spread from Wuhan, China, in December 2019 to a large number of countries within three months. The objective of this work is to report the initial
\end{abstract}

Correspondence: Dr Manas Kamal Sen, Department of Pulmonary, Critical Care and Sleep Medicine, Vardhman Mahavir Medical College and Safdarjung Hospital, New Delhi 110029, India. Tel. +91.09968306997. E-mail: drmksen@yahoo.com

Keywords: COVID-19; initial; mild.

Contributions: All the 20 authors contributed to the conception or design of the work or to the acquisition, analysis, and interpretation of data for the work. They also participated in the drafting the work or revised it critically for important intellectual content. They give their final approval of the version to be published, and agreed to be accountable for all aspects of the work in ensuring that questions related to the accuracy or integrity of any part of it are appropriately investigated and resolved.

Informed consent: The authors certify that they have obtained written informed consent from the patient to publish his personal details, images, surgical details and follow-up. The patient understands that his name and initials will not be published but anonymity cannot be guaranteed.

Financial support and sponsorship: None.

Conflict of interests: There is no conflict of interests

Place of study: Safdarjung Hospital and Vardhman Mahavir Medical College, New Delhi 110029, India.

Received for publication: 4 April 2020.

Accepted for publication: 6 April 2020.

${ }^{\circ}$ Copyright: the Author(s), 2020

Licensee PAGEPress, Italy

Monaldi Archives for Chest Disease 2020; 90:1294

doi: 10.4081/monaldi.2020.1294

This article is distributed under the terms of the Creative Commons Attribution Noncommercial License (by-nc 4.0) which permits any noncommercial use, distribution, and reproduction in any medium, provided the original author(s) and source are credited. experience with epidemiologic and clinical features, as well as with the management of COVID-19 patients in India. This is a descriptive case series of the first 21 COVID-19 infected patients confirmed with polymerase chain reaction (PCR) and admitted to a tertiary care centre in India from 01.02.2020 to 19.03.2020. Clinical, laboratory, and radiologic data were collected, including age, sex, nationality, travel history, symptoms, duration of stay, and comorbidities. The mean age of the population was 40.3 years with a male preponderance. Thirteen $(62 \%)$ patients had recent travel history outside India in the previous 30 days, two thirds of whom had travelled to Italy. The most common symptoms were fever and cough $(42.9 \%)$ followed by sore throat, headache and breathlessness. Vital and laboratory parameters were preserved in all patients and none of them required ventilatory support. Among the first 21 patients diagnosed with COVID-19 infection in India, the typical clinical presentation consisted in a mild upper respiratory tract infection predominantly affecting the young male population. One patient required supplemental oxygen. All patients recovered with no residual symptoms.

\section{Introduction}

The 2019 novel coronavirus (2019-nCoV) or COVID-19 as it is now called, is rapidly spreading worldwide from its place of origin in Wuhan City of Hubei Province of China [1]. Up to March $21^{\text {st }} 2020$ around 234,076 confirmed cases of coronavirus disease 2019 (COVID-19) and 9840 confirmed deaths have been reported from 176 countries, regions and territories [2]. India has reported 195 confirmed cases as of 20.03.2020. This is the third coronavirus that emerged among the human population in the last

\footnotetext{
*The Safdarjung Hospital COVID 2019 working group:

Nitesh Gupta, Sumita Agrawal, Pranav Ish, Suruchi Mishra, Rajni Gaind, Ganapathy Usha, Balvinder Singh, Manas Kamal Sen, Shibdas Chakrabarti (Consultant and Head, Pulmonary Medicine); NK Gupta (Professor, Pulmonary medicine); Dipak Bhattacharya (Consultant, Pulmonary medicine); Rohit Kumar (Assistant Professor, Pulmonary Medicine); Siddharth R. Yadav (Assistant Professor, Pulmonary Medicine); Rushika Saksena (Specialist, Microbiology); Rojaleen Das (Assistant Professor, Microbiology); Vikramjeet Dutta (Assistant Professor, Microbiology); Anupam Kr Anveshi (Senior Resident, Microbiology); Santvana Kohli (Assistant Professor, Anaesthesiology); Naveen KV(Assistant Professor, Anaesthesiology); Amandeep Jaswal (Assistant Professor, Anaesthesiology).
} 
two decades. The other two were the severe acute respiratory syndrome coronavirus (SARS-CoV) outbreak in 2002 and the Middle East respiratory syndrome coronavirus (MERS-CoV) outbreak in 2012 [2].

Human-to-human transmission via droplets as well as through contact with fomites seems to be the critical route of the virus spread. Since $80 \%$ of the infected population are either asymptomatic or have mild disease, people have been going to their workplaces and even traveling internationally. Nevertheless, even though the virus is causing mild disease in many, the course of illness may be severe, leading to hospitalization and even death in elderly or those with comorbid conditions [2].

Guan et al. published a report on 1099 patients with laboratoryconfirmed Covid-19 from 552 hospitals in 30 provinces, autonomous regions, and municipalities in mainland China through January 29, 2020. The most common symptoms reported were fever (43.8\% on admission, and $88.7 \%$ during hospitalization) and cough $(67.8 \%)$, diarrhoea $(3.8 \%)$ was uncommon. A severe form of the disease was reported in elderly and in patients with comorbidities. Overall reported cases of death in this study were 15 (1.4\%) [2].

The case reported from the United States was virus-positive in respiratory and faecal specimens; he had respiratory failure requiring oxygen supplementation. The patient was treated with intravenous Remdesivir (a novel nucleotide analogue prodrug under development) and reported to be afebrile and stable [3].

The objective of this case series from India is to describe the clinical characteristics at the early stage of the outbreak in India which experienced no previous outbreaks of coronavirus. We present data from a tertiary care setting in India used for isolation of suspected cases and management of COVID-19 positive patients. This is the first report from the Indian subcontinent.

\section{Methods}

Safdarjung Hospital is a tertiary care hospital in New Delhi and has been designated for isolation and management of suspected cases of COVID-19. The current study is a retrospective observational case series of epidemiological features and clinical manifestations of COVID-19 positive patients who presented themselves to this centre during the outbreak of 2019-2020 from $1^{\text {st }}$ February 2020 to $19^{\text {th }}$ March 2020.

Since the first reports of the outbreak of SARS-CoV-2 came from China, the Indian Ministry of Health and Family Welfare (MoHFW) implemented its preventive measures and management services for all suspected patients affected by the virus. According to safety criteria, in the presence of a suspect case at our centre, nursing staff take precautionary measures, such as medical PPE. International travellers and their contacts were placed in quarantine facilities. Individuals with symptoms or requiring hospitalization for any medical treatment, who met the criteria for a suspected case defined as 'symptomatic patient with travel history to countries with COVID-19 patients over the previous last 14 days, or who exposed themselves to a COVID-19 positive patient in the community', were admitted to our hospital. The isolation facility at our hospital was assessed for preparedness according to a checklist standardized by MoHFW [4]. All the health-care workers caring for infected patients received comprehensive training and demonstrated competence in implementing infection control practices and procedures. The nasopharyngeal and oropharyngeal swabs were tested at the National Centre for Disease Control (NCDC) for detection of COVID-19 using quantitative polymerase chain reaction for confirmation. All laboratory-confirmed cases were eligible for inclusion in this study and the epidemiological, clinical, and laboratory data were prospectively recorded.

\section{Results}

Twenty-one patients were included in the study over a period of time going from 1.2.2020 to 19.03.2020. The sociodemographic profile is summarized in Table 1. The mean age of participants was 40.3 years (range 16-73 years). There was a male preponderance (66.7\%). All patients were Indian. Seven were from Delhi, eight from Agra, two from Kolkata, and the remaining four were from Noida, Ghaziabad, Gurgaon, and Rajasthan, respectively.

The average length of stay in hospital was 11.54 days and the mean duration of symptoms was 2.1 days. Eleven (52.4\%) patients had a history of contact with a lab-confirmed COVID-19 patient. More than half of the patients, 13 (61.9\%), had a travel history outside India. The majority of patients $8(38.1 \%)$ visited Italy, followed by $2(9.5 \%)$ who travelled to London, and other 3 patients $(4.8 \%)$ who had been to Iran, Saudi Arabia, and Malaysia, respectively as shown in Figure 1.

The clinical and laboratory profile is summarized in Table 2. More than half $(57.1 \%)$ of the patients were symptomatic. The common presentation and symptoms experienced by nine patients were cough and fever. Sore throat, breathlessness, and headache were present in 5(23.8\%), 1(4.8\%), 3 (13.6\%), respectively.

Six patients $(28.6 \%)$ had comorbidities. The most common comorbidity was hypertension ( 5 patients) and diabetes mellitus ( 3 patients) adequately controlled with drugs. One (4.8\%) patient with hypertension and one with diabetes mellitus also showed anxiety disorder and hypothyroidism. Another patient had an underlying migraine and obstructive sleep apnoea.

Chest roentgenogram was performed in all patients at initial presentation, and in 20 cases (95.2\%), it showed no abnormality.

Table 1. Demographic profile of COVID-19 patients $(n=21)$.

\begin{tabular}{lcc}
\multicolumn{4}{c}{$\begin{array}{c}\text { Socio-demographic profile of patients } \\
\text { Frequency }(\mathrm{n}=21)\end{array}$} & $\%$ \\
\hline $\begin{array}{l}\text { Age group } \\
0-20 \text { years }\end{array}$ & 1 & 4.8 \\
$21-40$ & 10 & 47.6 \\
\hline $41-60$ & 8 & 38.1 \\
$>60$ & 2 & 9.5 \\
\hline Gender & & \\
Male & 14 & 66.7 \\
Female & 7 & 33.3 \\
\hline Nationality & & \\
Indian & 21 & 100 \\
Foreigner & 0 & 0 \\
\hline History of travel outside India in last 30 days & \\
Yes & 13 & 61.9 \\
No & 8 & 38.1 \\
\hline Family/office exposure to positive patients & \\
Family exposure & 8 & 38.1 \\
Work place exposure & 2 & 9.5 \\
\hline
\end{tabular}


Only one patient showed bilateral consolidation of lower lobes of lungs. There was no pleural effusion or lymphadenopathy. He was a 46-year-old male without comorbidities who required oxygen but no ventilatory support.

Mean saturation on room air (sp02) was 96.56\% (SD+2.13), and the respiratory rate was 18.19 (SD+3.02). Laboratory parameters were preserved with mean haemoglobin levels of 14.42 gram $\%$, leucocyte count of $5758 / \mathrm{mm}^{3}$, blood urea $23.56 \mathrm{mg} \%$, and serum bilirubin levels of $0.4875 \mathrm{mg} \%$. Only one patient had leukopenia with a count of $2900 / \mathrm{mm}^{3}$. Up to $19^{\text {th }}$ March 2020 , fifteen patients had been discharged and advised home quarantine for 14 days as per guidelines. All discharged patients became asymptomatic and tested negative twice according to the guidelines [4]. The mean duration for defervescence was $5 \pm 1.2$ days.

\section{Discussion}

The current series of 21 patients represents the first initial datum on coronavirus in India. The case series presents epidemiological and clinical characteristics of patients outside of China where the disease had originated. The infection was observed in both adolescents and elderly with a predominance of young adults. This may be related to limited travel by very young and elderly people and may not be related to susceptibility of these populations. The majority $(61.9 \%)$ of patients had history of international travel, most frequently to Italy. However, none of the cases reported travel history to China. Almost half (42.9\%) of patients were asymptomatic. An important thing to notice in published literature is that patients who develop respiratory failure rapidly deteriorate and do not respond to non-invasive ventilation [2,5]. In our study, one patient developed breathlessness that was managed conservatively with oxygen and supportive therapy; but none required invasive mechanical support. The most common symptoms were fever and dry cough. None of our patients had rhinitis or diarrhoea. The results of the present study are similar to initial data on 18 patients from Singapore, where most of them had fever and cough with infrequent rhinorrhoea. Only one patient in their cohort required ventilatory support, the rest of them responded with conservative management [6].

Table 2. Clinical and laboratory profile of COVID-19 patients.

\begin{tabular}{lcc} 
& n=21 & $\%$ \\
\hline Profile & 9 & 42.9 \\
Asymptomatic & 12 & 57.1 \\
Symptomatic & & \\
\hline Symptoms & 9 & 42.9 \\
Fever & 9 & 42.9 \\
Cough & 5 & 23.8 \\
\hline Sore throat & 3 & 13.6 \\
Headache & 1 & 4.8 \\
\hline Breathlessness & & \\
Comorbidities & 5 & 23.8 \\
Hypertension & 3 & 14.2 \\
Diabetes mellitus & 1 & 4.7 \\
\hline Anxiety & 1 & 4.7 \\
Hypothyroidism & 1 & 4.7 \\
\hline Migraine & 1 & 4.7 \\
Obstructive sleep apnoea & &
\end{tabular}

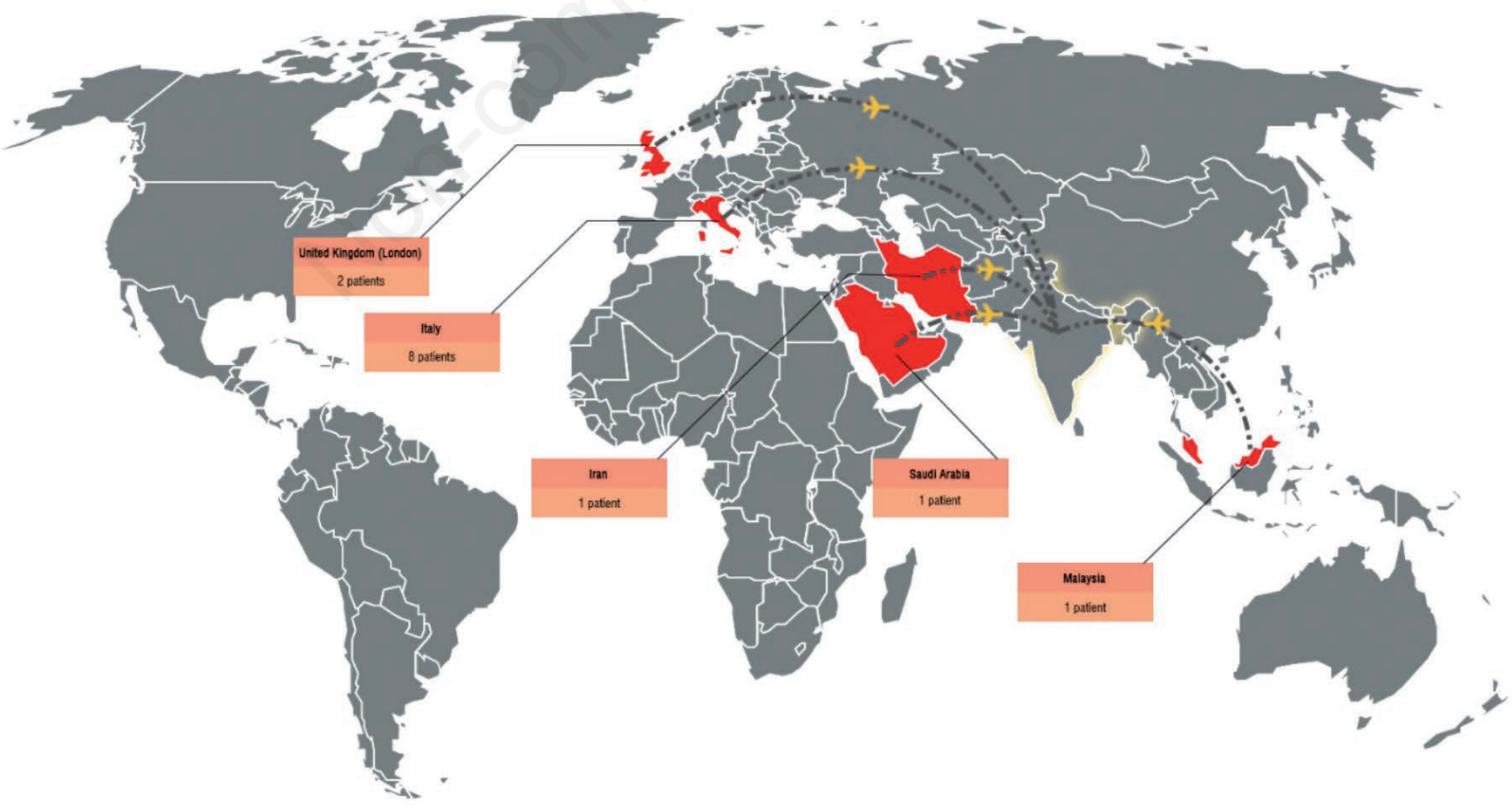

Figure 1. Thirteen patients who had international travel history. 
If we take into consideration the $\mathrm{H} 1 \mathrm{~N} 1$ pandemic phases given by WHO [7], the virus is currently in its phase $5 / 6$ of the pandemic in many parts of the world and, although today it seems like India is in phase 2, it will change soon into phase 3 if we do not contain it [4]. What it is important to be know is that as this disease is causing rapid respiratory failure in a handful of people, when we talk about a global perspective, the handful can mean a lot to deal with in terms of logistics and resources. Now that we have seen the initial pattern of the disease, we think it is time to come up with some guidelines for isolation, triage and treatment of these patients, both globally and locally. Studies are being conducted to identify the role of antiviral protease inhibitors against COVID-19. The Indian guidelines issued by the Indian Council of Medical Research allowed for the use of lopinavir and ritonavir for severe cases with respiratory failure or organ dysfunction [4]. However, a recent randomized trial has not found any benefit over time from the use of these drugs, both in terms of clinical improvement and mortality [8]. Chloroquine has also been evaluated in individual studies and may have a role in the management of COVID-19 in times to come [9]. Though initial results are not exciting, we shall continue with the search for effective treatment and vaccination [10]. Long-term sequelae like lung fibrosis can only be evaluated with serial followup of such patients. This study gives us some evidence to work on. First of all, preventing transmission is the key to curtail the pandemic. Our result showing a mild disease with complete recovery has been the similar initial trend seen in countries like Singapore and South Korea $[5,11]$. Since we are all struggling with resources, the isolation of all cases at a tertiary centre may not be feasible, 'self-isolation and frequent handwashing' should me the 'mantra' to be spread worldwide. Secondly, severe cases must undergo invasive mechanical ventilation early with supportive and conservative fluid strategy. Currently, no opinion is available on the use of antiviral drugs and we hope that the near future will provide us with trials able to answer this most awaited question. Thirdly, all countries have to use all available infrastructures strategically and to convert them into critical care facilities whenever it is required.

\section{Limitations}

The sample size in the current study is characterised by a small cohort. The spread of the pandemic to various geographic locations, age groups and comorbid patients may behave differently and hence these results may be difficult to generalize at national or international level. No drugs were used in any patient as therapy; hence, the role of protease inhibitors or chloroquine cannot be elucidated.

\section{Conclusions}

Among the first 21 patients diagnosed with COVID-19 admitted to a tertiary care hospital in India, the clinical presentation is of an upper respiratory tract infection which is mild, self-resolving with preserved vitals and organ-functions. These are encouraging observations which can be used to draft further national recommendations. The containment of spread is the most crucial determinant of the final morbidity from this COVID-19 pandemic. In the current series all patients recovered with no mortality. Although the virus is contagious and spreads globally, it is too early to comment on its virulence in India. Community transmission has not yet been reported at the time of publication. Follow-up of disease spread and clinical presentation in the larger population will give an insight into the COVID-19 outbreak in India. Close monitoring and large-scale control strategies will be needed to prevent widespread transmission within the community.

\section{References}

1. De Wit E, Van Doremalen N, Falzarano D, Munster VJ. SARS and MERS: recent insights into emerging coronaviruses. Nat Rev Microbiol 2016;523-34.

2. Guan W, Ni Z, Hu Y, et al. Clinical characteristics of coronavirus disease 2019 in China. N Engl J Med 2020. doi: 10.1056/NEJMoa2002032.

3. Holshue ML, DeBolt C, Lindquist S, et al. First case of 2019 novel coronavirus in the United States. N Engl J Med 2020;382:929-936.

4. Indian Council of Medical Research. Media report on "Briefing on COVID19". Accessed on: March 20, 2020. Available from https:/www.icmr.nic.in/sites/default/files/MediaReport_COVI D19.pdf

5. Rosenbaum L. Facing Covid-19 in Italy - Ethics, logistics, and therapeutics on the epidemic's front line. N Engl J Med 2020. doi: 10.1056/NEJMp2005492

6. Young BE, Ong SWX, Kalimuddin S, et al. Epidemiologic features and clinical course of patients infected with SARSCoV-2 in Singapore. JAMA 2020 doi: 10.1001/jama.2020.3204. [Epub ahead of print].

7. WHO. Current WHO phase of pandemic alert for Pandemic (H1N1) 2009. Accessed on: March 20, 2020. Available from: https://www.who.int/csr/disease/swineflu/phase/en/

8. Cao B, Wang Y, Wen D, et al. A trial of lopinavir-ritonavir in adults hospitalized with severe Covid-19. N Engl J Med 2020. doi: 10.1056/NEJMoa2001282 [Epub ahead of print].

9. Cortegiani A, Ingoglia G, Ippolito M, et al. A systematic review on the efficacy and safety of chloroquine for the treatment of COVID-19. J Crit Care 2020. pii: S0883-9441(20)30390-7. doi: 10.1016/j.jcrc.2020.03.005. [Epub ahead of print].

10. Baden LR, Rubin EJ. Covid-19 - The search for effective therapy. N Engl J Med 2020. doi: 10.1056/NEJMe2005477

11. COVID-19 National Emergency Response Center, Epidemiology and Case Management Team, Korea Centers for Disease Control and Prevention. Early epidemiological and clinical characteristics of 28 cases of coronavirus disease in South Korea. Osong Public Health Res Perspect 2020;11:8-14. doi: 10.24171/j.phrp.2020.11.1.03. 